\title{
Ovarian Cancer: Opportunity for Targeted Therapy
}

\author{
Tomoko Tagawa, ${ }^{1,2}$ Robert Morgan, ${ }^{1}$ Yun Yen, ${ }^{1}$ and Joanne Mortimer ${ }^{1}$ \\ ${ }^{1}$ Department of Medical Oncology, City of Hope National Medical Center, Duarte, CA 91010, USA \\ ${ }^{2}$ Department of Medical Oncology and Therapeutics Research, City of Hope National Medical Center, \\ 1500 E Duarte Rd, Duarte, CA 91010, USA
}

Correspondence should be addressed to Tomoko Tagawa, ttagawa@coh.org

Received 10 September 2011; Accepted 1 November 2011

Academic Editor: Ritu Salani

Copyright ( $) 2012$ Tomoko Tagawa et al. This is an open access article distributed under the Creative Commons Attribution License, which permits unrestricted use, distribution, and reproduction in any medium, provided the original work is properly cited.

\begin{abstract}
Ovarian cancer is a common cause of cancer mortality in women with limited treatment effectiveness in advanced stages. The limitation to treatment is largely the result of high rates of cancer recurrence despite chemotherapy and eventual resistance to existing chemotherapeutic agents. The objective of this paper is to review current concepts of ovarian carcinogenesis. We will review existing hypotheses of tumor origin from ovarian epithelial cells, Fallopian tube, and endometrium. We will also review the molecular pathogenesis of ovarian cancer which results in two specific pathways of carcinogenesis: (1) type I low-grade tumor and (2) type II high-grade tumor. Improved understanding of the molecular basis of ovarian carcinogenesis has opened new opportunities for targeted therapy. This paper will also review these potential therapeutic targets and will explore new agents that are currently being investigated.
\end{abstract}

\section{Introduction}

Ovarian cancer is the most common cause of gynecologic neoplasm and is the fifth cause of cancer mortality in women. The high mortality rate in women with epithelial ovarian cancer (EOC) is due to its detection at advanced stages. Even though there have been improvements in surgical techniques and treatment options, five-year survival for stage III and IV ovarian cancer still remains at approximately 45\% [1].

Known risk factors of EOC include nulliparity, early menarche, late menopause, and age. A particularly significant risk factor is a strong family history of breast and ovarian cancer. $10 \%-15 \%$ of women with ovarian cancer have genetic predispositions of BRCA1 and BRCA 2 mutations [2]. BRCA1 is associated with a $40 \%$ lifetime risk of ovarian cancer, and BRCA 2 has an approximately 15\% lifetime risk of ovarian cancer. Epidemiological studies show a reduction in the incidence of EOC in developed countries [2].

Part of the complexity of EOC lies in its heterogeneity. EOC can be classified into diverse group of tumors on the basis of morphology and molecular genetic features. This paper will review the current understanding of the molecular and morphologic heterogeneity of EOC as well as possible explanations of pathogenesis that contribute to the heterogeneity.

\section{Tumor Origin and Pathogenesis}

EOC origins are difficult to ascertain, because the majority of cases are diagnosed at late stages. Thus, there are limited records regarding early-stage disease. Historically, EOC is thought to originate from the ovarian epithelial surface and undergoes progressive dedifferentiation and spreads to the pelvic and abdominal cavities prior to metastasizing to distant organs $[2,3]$. However, EOC which predominantly consists of serous, endometrioid, and mucinous cell types is morphologically columnar and ciliated, similar to Mullerian epithelial cell lining of the endometrium, endocervix, fallopian tube, and gastrointestinal tract [3]. The ovarian epithelial surface, where these cells are purported to have originated from, consists of a single mesothelial layer of cells that are flattened and squamous-like. To explain this discrepancy, the traditional theories suggest that the mesothelial lining of the ovary invaginates to form paraovarian cysts that acquire 
Mullerian cell lining features and undergo malignant transformation [4]. The enlarging tumor envelops the ovary and is diagnosed as an adnexal mass of ovarian origin $[5,6]$.

Increasing evidence now suggests that the Fallopian tube may be an alternative site of tumor origin in many diagnosed as primary EOC [5]. In older studies, the origin of EOCs were presumed to be the ovaries, and Fallopian tubes were typically not examined. However, more recently, observational studies have shown that in situ and early invasive tubal carcinomas occur in women with a genetic predisposition for ovarian cancer $[5,7,8]$. Furthermore, over $70 \%$ of nonhereditary ovarian cancer and peritoneal highgrade serous carcinomas revealed serous epithelial carcinoma in the Fallopian tube and mucosal tubal involvement [9].

The fimbria of the Fallopian tube are abundant with angiolymphatic vasculature and are in direct contact with the basement membrane of the Fallopian tube. Through this vasculature, the serous tubal intraepithelial carcinoma may conceivably disseminate to the surface of the ovary and peritoneum without invasive growth from the Fallopian tube $[10,11]$. Therefore, rather than the tumor originating from a cyst that developed from the mesothelial lining of the ovary, tubal epithelium may directly implant into the surface of the ovary to form an inclusion cyst which subsequently develops into tubal epithelial carcinoma $[3,10]$. An alternative possibility is that normal tubal intraepithelial cells implant into the ovary at the time of ovulation and develop into inclusion cysts that transform into carcinoma over time $[4,10]$.

Similarly, the endometrioid and clear cell carcinomas are thought to originate from endometriosis. According to this theory, endometrial cells "escape" the uterus via retrograde menstrual flow and implant the ovary or pelvic cavity secondarily. This mechanism has been supported by multiple morphologic and molecular studies [12, 13].

\section{Morphologic and Molecular Characteristics}

EOC was initially categorized into invasive serous carcinoma and serous borderline tumor (SBT) which was defined as a low malignant potential carcinoma lacking invasive growth. More recently, SBT was further subdivided into (1) atypical proliferative serous tumor (APST) and (2) micropapillary serous carcinoma (MPSC), a possible precursor to low-grade serous carcinoma (LGSC) [10]. Previously, serous carcinoma was thought be a spectrum of disease, where LGSC progressed to high-grade serous carcinoma (HGSC) over time. However, with the understanding that LGSC arises from SBT, high-grade invasive carcinoma is thought to be a disparate entity. This resulted in a model of ovarian carcinogenesis that consists of two distinct pathways and subtypes $[3,4,14]$.

The two subtypes are low-grade (type I) and high-grade (type II). Type I tumors are typically indolent, slow growing tumors that are often detected at early stages. Type I tumors include low-grade serous, low-grade endometrioid, clear cell, and mucinous carcinomas $[15,16]$. Type II includes highgrade serous, high-grade endometrioid, mixed mesodermal (carcinosarcoma), and undifferentiated carcinomas. Type II tumors are typically diagnosed in advanced stages. The majority of EOC, approximately 75\%, are type II aggressive tumors $[17,18]$. Type I tumors have a median survival of 81 months compared to 57 to 65 months in type II tumors.

Molecular and genetic differences are now being recognized to further understand the distinction between these two subtypes. The main genetic difference between the two subtypes that explains duality in their malignant potentials is (1) type I tumors have genetically stable and isolated mutations and (2) type II tumors have significant genetic instability and involve p53 mutations that result in a more aggressive and invasive phenotypic expression [4].

Mutational analysis and genetic expression studies have shown that APST, MPSC, and LGSC share molecular mutations that are significantly different from molecular alterations in HGSC [10]. Type I tumors typically involve mutations in a number of genes such as KRAS, BRAF, PTEN, PIK3-CA, and CTNNB1 which encodes beta-catenin [10, 14]. Mutations in these upstream regulators result in constitutive activation of the MAPK signaling pathway. Up to $70 \%$ of MPSC and LGSC have been shown to express active MAPK signaling $[4,15]$. Her2/neu mutations have been detected in approximately $10 \%$ of Type I tumors and appear to be mutually exclusive with having KRAS and BRAF mutations $[10,15]$. Type I tumors rarely express p53 mutations. Furthermore, to further explain the phenotypic association between LGSC and MPSC that are not shared with APST, MPSC is more molecularly similar to LGSC than APST. On the basis of mutational analysis of clear cell carcinomas, the PI3K/PTEN pathway appears to be the most commonly deregulated [19]. While PTEN mutations are present in low-grade endometrioid histologies, they also have alterations in the $\mathrm{Wnt} / \beta$-catenin pathways and CTNNB1 mutations [20]. Therefore, among the type I tumor subtypes, variable genetic alterations have been identified that explain their phenotypic differences.

In contrast, type II tumors have TP 53 mutations in up to $95 \%$ of the cases. They are also characterized by genetic instability and high frequencies of DNA copy number gains and losses. They rarely contain KRAS and BRAF mutations. A recent publication in Nature identified 9 significantly mutated genes in high-grade serous ovarian cancer. The most common were RB1 mutations (67\%), TP 53 mutations (96\%), PI3/Ras pathways (47\%), and BRCA 1 or 2 mutations (22\%) [21]. De novo, nonfamilial cases of BRCA1 and BRCA2 inactivation are often associated with hypermethylation. In a genomic analysis of high-grade ovarian cancer, $11 \%$ of BRCA1 silencing was the result of hypermethylation and epigenetic modification rather than mutations. Studies have shown that mutated or hypermethylated BRCA carriers respond to PARP inhibitor therapy $[22,23]$.

\section{MicroRNA}

MicroRNAs are short nucleotide sequences that are noncoding RNAs that are involved in the regulation of posttranscriptional genes. MicroRNAs are critical in cell development but may also contribute to tumor origin [24-27]. MicroRNAs have been found to be differentially expressed between ovarian carcinoma compared to normal ovarian epithelial cells [28]. Upregulation or downregulation of microRNA 
that regulate oncogenes and tumor suppressor genes, respectively, can induce malignant transformation. Dysregulation of microRNA expression has been associated with highgrade serous ovarian carcinoma. The relationship between such microRNA dysregulation and BRCA 1 or 2 expression is being explored [28]. Furthermore, microRNA have previously been found to regulate cellular differentiation $[29,30]$ and may play a role in epithelial to mesenchymal cell transformation during carcinogenesis of ovarian epithelial cells. Exploration of microRNA involvement in ovarian carcinogenesis has recently focused attention on Let7 microRNA and HMGA2 gene regulation.

\section{HMGA2 and Let-7}

HMGA2, a high-mobility-group AT-hook protein, is a nonhistone DNA-binding factor that attaches to AT-rich sequences in the minor groove of the DNA helix. It is an important regulator of cell growth, differentiation, apoptosis, and malignant transformation. HMGA2 expression is regulated by the microRNA Let-7. Preclinical studies have evaluated the role of let- 7 as a tumor suppressor gene to the oncogenic mechanism of HMGA2 [31]. When the let-7 activity is downregulated, HMGA2 activity is no longer repressed and contributes to malignant transformation [31]. HMGA2 overexpression has been identified in $65 \%$ of ovarian carcinoma but are rarely expressed in normal ovarian epithelial cells [32-34]. Furthermore, HMGA2 overexpression has been associated with high-grade serous ovarian carcinoma $[32,33]$. The Let-7 HMGA2 dysregulation may be a key factor in ovarian carcinogenesis distinguishing low-grade or type I from high-grade or type II EOC [35].

In our institution, we compared the HMGA2 expression in serous OC and endometrioid OC surgical specimens. In this study, we attempted to distinguish expression patterns on the basis of the phenotypic grade of the OC. Fourteen consecutive endometrioid OC were analyzed and twelve consecutive high-grade serous carcinomas were analyzed for HMGA2 expression by immunohistochemical staining. High-grade serous carcinoma was associated with greater HMGA2 expression compared to endometrioid OC. Longitudinal evaluation is pending to evaluate the prognostic significance of HMGA2 expression and clinical outcome [41].

Other studies have further evaluated the correlation of HMGA2 overexpression and p-53 mutations [32] further demonstrating the tumorigenesis of type II high-grade EOC that is distinct from type I low-grade EOC. Furthermore, animal models have shown that HMGA2 silencing is associated with reduction in tumor growth and increased apoptosis of tumor cells [34]. This suggests that HMGA2 may be a possible target in ovarian cancer therapy.

\section{Opportunities for Targeted Therapy}

Despite optimal surgical and cytotoxic treatment of advanced ovarian cancer, only $10 \%$ to $15 \%$ achieve longterm remission, and the majority will face recurrent disease $[42,43]$. While there is a role for chemotherapy in recurrent disease, the effects are often short lived due to development of chemoresistance [44]. Targeted therapies in ovarian cancer are currently being investigated to find novel ways to overcome chemoresistance. These molecular targets of therapy include: VEGF (antiangiogenesis), EGFR tyrosine kinase, HER2 receptor, PARP, and MAPK/BRAF/MEK pathways (See Table 1).

\section{Antiangiogenesis}

Angiogenesis has been shown to be a key component in ovarian cancer metastasis and ascites development [45]. Vascular endothelial growth factor (VEGF) is a critical regulator of angiogenesis and is involved in various aspects of ovarian carcinogenesis [46]. Antiangiogenic therapy has been shown to have activity in ovarian cancer. Bevacizumab is a monoclonal antibody that targets VEGF-A. Two phase II trials with single-agent bevacizumab have shown $16 \%$ to $21 \%$ response $[47,48]$. Phase III studies involving chemotherapy with or without bevacizumab presented during the 2011 Annual Meeting of the American Society of Clinical Oncology (ASCO) showed a trend toward an overall survival benefit in patients treated with bevacizumab in addition to chemotherapy in the first line setting with $\mathrm{HR}=$ $0.64 P=0.0022$ in those with poor prognosis disease (ICON7). Similar data were reported in patients treated for platinum sensitive recurrent ovarian cancer with median overall survival of 35.5 months (+bevacizumab) versus 29.9 months (-bevacizumab) and $\mathrm{HR}=0.751 \mathrm{P}=0.094$ (OCEANS) [49].

Mammalian target of rapamycin (mTOR) is involved in cell growth and proliferation and induces increases in VEGF and platelet derived growth factor (PDGF) that ultimately activate angiogenesis. mTOR inhibitors have also shown single agent activity in the treatment of clear cell ovarian carcinoma [50]. The antiangiogenic effects are thought to be synergistic with that of bevacizumab. Results of a phase II study combining the mTOR inhibitor, temsirolimus, with bevacizumab suggest possible synergy [49]. Other phase I and II trials involving antiangiogenic therapy are showing activity as single agents or synergistically with chemotherapy. These agents include (1) VEGF trap (Aflibercept) which is a fusion protein that acts as a high-affinity VEGF receptor blocker, (2) Sunitinib (a PDGF and VEGF receptor inhibitor), (3) Vatalanib (a pan-VEGF receptor inhibitor), (4) Motesanib (a multikinase inhibitor of PDGF, VEGF, and cKIT), and (5) Cedarinib (a VEGF tyrosine kinase receptor inhibitor).

\section{Anti-HER2 Therapy}

Her2 expression in ovarian cancer has been variable. Many studies evaluate HER2 expression in ovarian cancer as a positive or negative expression and do not describe the staining intensity to characterize the overexpression of HER2 [51]. The studies that have evaluated HER2 overexpression demonstrate significant variability in intense HER2 staining from $1.8 \%$ to $35 \%[43,51-53]$. Studies have suggested that gene amplification does not always correlate with HER2 
TABLE 1: Potential targets of therapy in ovarian cancer.

\begin{tabular}{|c|c|c|c|}
\hline Targets & Inhibitors & Studies & NCI study \\
\hline \multirow{4}{*}{ VEGF } & \multirow{4}{*}{ Bevacizumab } & \multirow{4}{*}{ Phase III [49] } & GOG 218 \\
\hline & & & ICON 7 \\
\hline & & & GOG 213 \\
\hline & & & OCEANS \\
\hline \multirow{23}{*}{ VEGF TKI } & \multirow{3}{*}{ Sunitinib } & \multirow{3}{*}{ Phase II } & NCT00979992 \\
\hline & & & NCT00768144 \\
\hline & & & NCT00388037 \\
\hline & Vatalanib & Phase I (+doce) & NCT00268918 \\
\hline & \multirow{6}{*}{ Sorafenib } & Phase II (+carb/pacli) & NCT00390611 \\
\hline & & Phase II (+topo) & NCT01047891 \\
\hline & & Phase II & NCT00522301 \\
\hline & & Phase II (+bev) & NCT00436215 \\
\hline & & Phase II [36] & NCT00093626 \\
\hline & & Phase II (+carb/pacli) & NCT00096200 \\
\hline & \multirow{3}{*}{ Vandetanib } & Phae I/II (+bortezomib) & NCT00923247 \\
\hline & & Phase II & NCT00445549 \\
\hline & & Phase II(+/- doce $)$ & NCT00872989 \\
\hline & \multirow{3}{*}{ Cediranib } & Phase I/II(+olaparib) & NCT01116648 \\
\hline & & Phase II & NCT00275028 \\
\hline & & Phase II (+temsirolimus) & NCT01065662 \\
\hline & \multirow{5}{*}{ Pazopanib } & Phase I/II (+cyclophosphamide) & NCT01238770 \\
\hline & & Phase II & NCT01262014 \\
\hline & & Phase I/II [37] & NCT01035658 \\
\hline & & Phase II & NCT00281632 \\
\hline & & Phase III & NCT00866697 \\
\hline & Vargatef & Phase I (+everolimus) & Pending \\
\hline & AMG 706 & Phase II & NCT00574951 \\
\hline \multirow{2}{*}{ VEGF TRAP } & \multirow{2}{*}{ Aflibercept } & Phase II & NCT00327171 \\
\hline & & Phase II (+doce) & NCT00436501 \\
\hline \multirow{10}{*}{ PI3K-PTEN-A } & \multirow{5}{*}{ Temsirolimus } & Phase I (+lip Doxo) & NCT00982631 \\
\hline & & Phase II & NCT00926107 \\
\hline & & Phase II (carb/pacli) & NCT01196429 \\
\hline & & Phase II & NCT00429793 \\
\hline & & Phase I/II(+bev) & NCT01010126 \\
\hline & \multirow{4}{*}{ Everolimus } & & \\
\hline & & Phase II (+bev) & NCT01031381 \\
\hline & & Phase II (+/- bev) & NCT00886691 \\
\hline & & Phase II (+lip doxo) & NCT01281514 \\
\hline & Ridaforolimus & Phase I (+carb/pacli) & NCT01256268 \\
\hline
\end{tabular}


Table 1: Continued.

\begin{tabular}{|c|c|c|c|}
\hline Targets & Inhibitors & Studies & NCI study \\
\hline \multirow{10}{*}{ EGFR } & \multirow{2}{*}{ Cetuximab } & Phase II (carb/pacli) & NCT00063401/ \\
\hline & & Phase II (+carb) & NCT00086892 \\
\hline & \multirow{5}{*}{ Erlotinib } & Phase II (+bev) [38] & NCT00130520 \\
\hline & & Phase II (+topo) & NCT01003938 \\
\hline & & Phase I/II (+carb/pacli) & NCT00217529 \\
\hline & & Phase II (+carb) & NCT00030446 \\
\hline & & Phase II (+bev after carb/pacli/bev) & NCT00520013 \\
\hline & \multirow{3}{*}{ Lapatinib } & Phase II (+topo) [39] & NCT00436644 \\
\hline & & Phase II & NCT00113373 \\
\hline & & Phase I/II (+carb/pacli) & NCT00316407 \\
\hline \multirow{4}{*}{ HER2 } & Trastuzumab & Phase II [40] & \\
\hline & \multirow{2}{*}{ Pertuzumab } & Phase II & NCT00058552 \\
\hline & & Phase II (+gemcitabine) & NCT00096993 \\
\hline & Lapatinib & See above & \\
\hline \multirow{13}{*}{ PARP } & \multirow{4}{*}{ ABT 888} & Phase II (+temoz versus lip doxo) & NCT01113957 \\
\hline & & Phase II (+cyclophosphamide) & NCT01306032 \\
\hline & & Phase I/II (+topo) & NCT01012817 \\
\hline & & Phase I (+carb/pacli/bev) & NCT00989651 \\
\hline & \multirow{7}{*}{ Olaparib } & Phase II (+carb/pacli) & NCT01081951 \\
\hline & & Phase I (+carb) & NCT00647062 \\
\hline & & Phase II & NCT00679783 \\
\hline & & Phase II & NCT00494442 \\
\hline & & Phase II (versus lip doxo) & NCT00628251 \\
\hline & & Phase II & NCT00753545 \\
\hline & & Phase II & NCT01033123 \\
\hline & \multirow{2}{*}{ Iniparib* } & Phase II (carb/gemcitabine) & NCT01033292 \\
\hline & & Phase II & NCT00677079 \\
\hline \multirow{3}{*}{ Epigenetic } & \multirow{2}{*}{ Decitabine } & Phase II (+carb) & NCT00477386 \\
\hline & & Phase I (+doxorubicin/vaccine) & NCT00887796 \\
\hline & Belinostat & Phase I/II (+carb or pacli) & NCT00421889 \\
\hline MAPK/RAF/MEK pathway & Cabozantinib & Phase I & NCT00940225 \\
\hline HMGA2 & Let-7 microRNA & Preclinical & \\
\hline
\end{tabular}

* Iniparib as a true PARP inhibitor is currently under investigation.

Doce: docetaxel, Carb: carboplatin, pacli: paclitaxel, topo: topotecan, bev: bevacizumab, temoz: temozolomide, and lip doxo: liposomal doxorubicin.

protein overexpression [54]. Furthermore, some data suggest that HER2 overexpression is mostly found in high-grade serous histology as opposed to low-grade endometrioid [55]. Her2 positive expression has demonstrated association with survival in patients with EOC [53, 56-58]. More recently, HER2 gene status was evaluated from the GINECO study, and no survival differences were detected in patients with or without HER2 overexpression who were treated with carboplatin/paclitaxel [59]. This suggests an association between HER2 status and paclitaxel sensitivity.
As a result of such variability in HER2 expression in ovarian cancer, the role of anti-HER2 therapy is unclear. Anti-HER2 therapy with trastuzumab and pertuzumab have shown modest activity in ovarian cancer [40,60]. Preclinical studies initially suggested activity in tumors without HER2 overexpression [61]. However, subset analyses of phase II trials indicate that pertuzumab has better activity in those with HER2 overexpression [60]. HER2 expression in EOC has not been studied as extensively as in breast cancer, and there are many inconsistent data. Thus, the limitations to 
anti-HER2 therapy hinges on further careful examination of HER2 oncogene as a potential prognostic, predictive, and therapeutic target.

\section{EGFR Tyrosine Kinase Inhibitors}

EGFR tyrosine kinase inhibitors are also being explored including cetuximab, lapatinib, and erlotinib [62, 63]. A phase II study of erlotinib with carboplatin has shown activity in platinum sensitive recurrent ovarian cancer $57 \%$ objective response rate in platinum sensitive and $7 \%$ in platinum-resistant patients) [64]. A phase II study with erlotinib, carboplatin, and paclitaxel in the first line setting showed no statistically significant pathologic complete response rates compared to historical controls [65]. A Phase I/II study of lapatinib with carboplatin and paclitaxel in recurrent stage III or IV ovarian and breast cancer proved safe with favorable response rates [66]. Recently, a phase II study was published which explored lapatinib combined with topotecan in platinum refractory or resistant ovarian cancer. This study showed minimal clinical activity with significant hematologic grade 3 and 4 toxicities [39]. A Phase II study with cetuximab and carboplatin in EGFR-positive ovarian cancer showed modest activity where 9 of 28 patients achieved objective response and 8 of 28 patients had stable disease [67]. Toxicities in this study included acneiform rash and hypersensitivity reactions. Another phase II study with cetuximab, carboplatin, and paclitaxel failed to demonstrate progression-free survival benefit compared to historical data [62].

\section{PARP Inhibitors}

Poly (ADP-ribose) polymerase (PARP) inhibitors block base excision repair. For tumors that lack DNA repair mechanisms due to BRCA1/BRCA2, HNPCC, Fanconi Anemia, and other genetic mutations, inhibiting alternate repair pathways with PARP inhibition may increase antitumor selectivity and improve chemotherapy sensitivity. Three phase II studies with PARP inhibitors, olaparib and iniparib, show activity in recurrent platinum sensitive ovarian cancer in combination with chemotherapy or as single-agent maintenance after chemotherapy [49]. Olaparib as a maintenance therapy has also been shown to improve progression-free survival by 3.6 months compared to placebo in platinum-sensitive relapsed serous ovarian cancer. (ASCO 2011 abstract 5003) There is currently an ongoing NCI sponsored randomized Phase II study that explores ABT 888 (veliparib) with cyclophosphamide in BRCA positive ovarian and triple negative breast cancer [68].

\section{Epigenetic Studies}

The pathophysiology of cancer is not only the result of inherited or sporadic genetic mutations but is also the result of epigenetic modifications in the genome. Histone hypoacetylation and abnormal DNA methylation also contribute to tumorigenesis and chemotherapy resistance. A phase II study with the DNA hypomethylating agent, decitabine, showed improved response rates in platinum-resistant ovarian cancer when added to carboplatin therapy at low dosages, where 9 out of 17 patients had progression-free survival at 6 months (ASCO 2011 abstract 5011) [49]. Other studies with the histone deacetylase inhibitor, belinostat, and the proteosome inhibitor, carfilzomib, are being explored in ongoing phase II trials.

\section{MAPK/BRAF/MEK Pathway}

One of the major pathways that regulate cellular growth is extracellular-related kinase (ERK) which triggers a cell surface-receptor mediated signaling cascade involving Ras, Raf, MEK [mitogen-activated protein (MAP)/ERK kinases] and ERK. As described previously, BRAF mutations are commonly associated with low-grade ovarian carcinoma. BRAF mutations result in the constitutive activation of the MAP kinase/MEK pathways. Furthermore, preclinical models have shown that in addition to BRAF mutations in low-grade tumors, Raf-1 isoform predominantly mediates ovarian cancer cell growth compared with Raf-A or B-Raf isoforms [69]. There have also been reports demonstrating reduced survival in ovarian cancer patients with increased expression of Raf-1 [70]. Thus, the MAPK/BRAF/MEK pathway can be a target in both high-grade and low-grade ovarian cancer. MET tyrosine kinase cell surface receptor is involved in RAF and MAP kinase activation pathways, and its inhibition results in downstream suppression of RAF and MAP kinase activity. The potent MET inhibitor, cabozantinib, showed activity in advanced ovarian cancer irrespective of platinum sensitivity [49].

\section{Let-7 MicroRNA Therapy}

Among the emerging next-generation therapies are the microRNA therapeutics. Preclinical mouse models with exogenous Let-7 microRNA have shown suppression of cell proliferation in breast cancer cells $[71,72]$. There are significant limitations to clinical applicability of microRNA technology at this time due to a limited understanding of the Let-7 mechanism and with methods of delivery.

\section{Conclusion}

Epithelial ovarian carcinoma is a highly heterogeneous disease process that is associated with significant mortality and morbidity. Recent progress in molecular characteristics of ovarian cancer has helped delineate the origins of carcinogenesis, particularly, a model of tumorigenesis which is based on a dichotomous theory of (1) low-grade or type I ovarian cancer associated with gene stability and multiple isolated mutations and (2) high-grade type II ovarian cancer associated with genetic instability and p53 mutations. Continued evaluation of the molecular makeup of ovarian carcinoma is critical in the further identification of treatment targets and improved clinical outcome. 


\section{Acknowledgment}

The project described was supported by Grant no. P30 CA033572 from the National Cancer Institute. Its contents are solely the responsibility of the authors and do not necessarily represent the official views of the National Cancer Institute or NIH.

\section{References}

[1] A. Jemal, R. Siegel, E. Ward, T. Murray, J. Xu, and M. J. Thun, "Cancer statistics, 2007," Ca-A Cancer Journal for Clinicians, vol. 57, no. 1, pp. 43-66, 2007.

[2] B. T. Hennessy, R. L. Coleman, and M. Markman, "Ovarian cancer," The Lancet, vol. 374, no. 9698, pp. 1371-1382, 2009.

[3] R. J. Kurman and I. M. Shih, "The origin and pathogenesis of epithelial ovarian cancer: a proposed unifying theory," American Journal of Surgical Pathology, vol. 34, no. 3, pp. 433 443, 2010.

[4] R. J. Kurman and I. M. Shih, "Pathogenesis of ovarian cancer: lessons from morphology and molecular biology and their clinical implications," International Journal of Gynecological Pathology, vol. 27, no. 2, pp. 151-160, 2008.

[5] J. R. Chien, G. Aletti, D. A. Bell, G. L. Keeney, V. Shridhar, and L. C. Hartmann, "Molecular pathogenesis and therapeutic targets in epithelial ovarian cancer," Journal of Cellular Biochemistry, vol. 102, no. 5, pp. 1117-1129, 2007.

[6] D. A. Bell, "Origins and molecular pathology of ovarian cancer," Modern Pathology, vol. 18, supplement 2, pp. S19-S32, 2005.

[7] J. M. Piek, P. J. van Diest, R. P. Zweemer et al., "Dysplastic changes in prophylactically removed Fallopian tubes of women predisposed to developing ovarian cancer," Journal of Pathology, vol. 195, no. 4, pp. 451-456, 2001.

[8] M. J. Callahan, C. P. Crum, F. Medeiros et al., "Primary fallopian tube malignancies in BRCA-positive women undergoing surgery for ovarian cancer risk reduction," Journal of Clinical Oncology, vol. 25, no. 25, pp. 3985-3990, 2007.

[9] D. W. Kindelberger, Y. Lee, A. Miron et al., "Intraepithelial carcinoma of the fimbria and pelvic serous carcinoma: evidence for a causal relationship," American Journal of Surgical Pathology, vol. 31, no. 2, pp. 161-169, 2007.

[10] I. M. Shih and R. J. Kurman, "Ovarian tumorigenesis: a proposed model based on morphological and molecular genetic analysis," American Journal of Pathology, vol. 164, no. 5, pp. 1511-1518, 2004.

[11] J. D. Seidman and F. Khedmati, "Exploring the histogenesis of ovarian mucinous and transitional cell (Brenner) neoplasms and their relationship with walthard cell nests: a study of 120 tumors," Archives of Pathology and Laboratory Medicine, vol. 132, no. 11, pp. 1753-1760, 2008.

[12] E. Veras, T. L. Mao, A. Ayhan et al., "Cystic and adenofibromatous clear cell carcinomas of the ovary: distinctive tumors that differ in their pathogenesis and behavior: a clinicopathologic analysis of 122 cases," American Journal of Surgical Pathology, vol. 33, no. 6, pp. 844-853, 2009.

[13] D. T. Wheeler, K. A. Bell, R. J. Kurman, and M. E. Sherman, "Minimal uterine serous carcinoma: diagnosis and clinicopathologic correlation," American Journal of Surgical Pathology, vol. 24, no. 6, pp. 797-806, 2000.

[14] R. J. Kurman and I.-M. Shih, "Molecular pathogenesis and extraovarian origin of epithelial ovarian cancer-Shifting the paradigm," Human Pathology, vol. 42, no. 7, pp. 918-931, 2011.

[15] I. M. Shih and R. J. Kurman, "Molecular pathogenesis of ovarian borderline tumors: new insights and old challenges," Clinical Cancer Research, vol. 11, no. 20, pp. 7273-7279, 2005.

[16] D. M. Gershenson, C. C. Sun, K. H. Lu et al., "Clinical behavior of stage II-IV low-grade serous carcinoma of the ovary," Obstetrics and Gynecology, vol. 108, no. 2, pp. 361-368, 2006.

[17] D. A. Bell and R. E. Scully, "Early de novo ovarian carcinoma. A study of fourteen cases," Cancer, vol. 73, no. 7, pp. 18591864, 1994.

[18] G. Singer, R. Stöhr, L. Cope et al., "Patterns of p53 mutations separate ovarian serous borderline tumors and lowand high-grade carcinomas and provide support for a new model of ovarian carcinogenesis: a mutational analysis with immunohistochemical correlation," American Journal of Surgical Pathology, vol. 29, no. 2, pp. 218-224, 2005.

[19] S. Jones, T. L. Wang, I. M. Shih et al., "Frequent mutations of chromatin remodeling gene ARID1A in ovarian clear cell carcinoma," Science, vol. 330, no. 6001, pp. 228-231, 2010.

[20] K. C. Wiegand, S. P. Shah, O. M. Al-Agha et al., "ARID1A mutations in endometriosis-associated ovarian carcinomas," New England Journal of Medicine, vol. 363, no. 16, pp. 15321543, 2010.

[21] Network TCGAR, "Integrated genomic analyses of ovarian cancer," Nature, vol. 474, pp. 609-615, 2011.

[22] P. C. Fong, D. S. Boss, T. A. Yap et al., "Inhibition of poly(ADPribose) polymerase in tumors from BRCA mutation carriers," New England Journal of Medicine, vol. 361, no. 2, pp. 123-134, 2009.

[23] J. Veeck, S. Ropero, F. Setien et al., "BRCA1 CpG island hypermethylation predicts sensitivity to poly(adenosine diphosphate)-ribose polymerase inhibitors," Journal of Clinical Oncology, vol. 28, no. 29, pp. e563-e564, 2010.

[24] R. I. Gregory and R. Shiekhattar, "MicroRNA biogenesis and cancer," Cancer Research, vol. 65, no. 9, pp. 3509-3512, 2005.

[25] E. Hernando, "microRNAs and cancer: role in tumorigenesis, patient classification and therapy," Clinical and Translational Oncology, vol. 9, no. 3, pp. 155-160, 2007.

[26] J. Winter and S. Diederichs, "MicroRNA biogenesis and cancer," Methods in Molecular Biology, vol. 676, pp. 3-22, 2011.

[27] W. Zhang, J. E. Dahlberg, and W. Tam, "MicroRNAs in tumorigenesis: a primer," American Journal of Pathology, vol. 171, no. 3, pp. 728-738, 2007.

[28] C. H. Lee, S. Subramanian, A. H. Beck et al., "MicroRNA profiling of BRCA1/2 mutation-carrying and non-mutationcarrying high-grade serous carcinomas of ovary," PLoS ONE, vol. 4, no. 10, Article ID e7314, 2009.

[29] M. Lin, W. Chen, J. Huang et al., "MicroRNA expression profiles in human colorectal cancers with liver metastases," Oncology Reports, vol. 25, no. 3, pp. 739-747, 2011.

[30] J. Lu, G. Getz, E. A. Miska et al., "MicroRNA expression profiles classify human cancers," Nature, vol. 435, no. 7043, pp. 834-838, 2005.

[31] C. Mayr, M. T. Hemann, and D. P. Bartel, "Disrupting the pairing between let-7 and Hmga2 enhances oncogenic transformation," Science, vol. 315, no. 5818, pp. 1576-1579, 2007.

[32] A. Mahajan, Z. Liu, L. Gellert et al., "HMGA2: a biomarker significantly overexpressed in high-grade ovarian serous carcinoma," Modern Pathology, vol. 23, no. 5, pp. 673-681, 2010.

[33] J. J. Wei, J. Wu, C. Luan et al., "HMGA2: a potential biomarker complement to P53 for detection of early-stage high-grade 
papillary serous carcinoma in fallopian tubes," American Journal of Surgical Pathology, vol. 34, no. 1, pp. 18-26, 2010.

[34] A. Malek, E. Bakhidze, A. Noske et al., "HMGA2 gene is a promising target for ovarian cancer silencing therapy," International Journal of Cancer, vol. 123, no. 2, pp. 348-356, 2008.

[35] S. Shell, S. M. Park, A. R. Radjabi et al., "Let-7 expression defines two differentiation stages of cancer," Proceedings of the National Academy of Sciences of the United States of America, vol. 104, no. 27, pp. 11400-11405, 2007.

[36] D. Matei, M. W. Sill, H. A. Lankes et al., "Activity of sorafenib in recurrent ovarian cancer and primary peritoneal carcinomatosis: a Gynecologic Oncology Group trial," Journal of Clinical Oncology, vol. 29, no. 1, pp. 69-75, 2011.

[37] M. Friedlander, K. C. Hancock, D. Rischin et al., "A phase II, open-label study evaluating pazopanib in patients with recurrent ovarian cancer," Gynecologic Oncology, vol. 119, no. 1, pp. 32-37, 2010.

[38] S. K. Chambers, M. C. Clouser, A. F. Baker et al., "Overexpression of tumor vascular endothelial growth factor A may portend an increased likelihood of progression in a phase II trial of bevacizumab and erlotinib in resistant ovarian cancer," Clinical Cancer Research, vol. 16, no. 21, pp. 5320-5328, 2010.

[39] S. J. Weroha, A. L. Oberg, K. L. Ziegler et al., "Phase II trial of lapatinib and topotecan (LapTop) in patients with platinumrefractory/resistant ovarian and primary peritoneal carcinoma," Gynecologic Oncology, vol. 122, no. 1, pp. 116-120, 2011.

[40] M. A. Bookman, K. M. Darcy, D. Clarke-Pearson, R. A. Boothby, and I. R. Horowitz, "Evaluation of monoclonal humanized anti-HER2 antibody, trastuzumab, in patients with recurrent or refractory ovarian or primary peritoneal carcinoma with overexpression of HER2: a phase II trial of the Gynecologic Oncology Group," Journal of Clinical Oncology, vol. 21, no. 2, pp. 283-290, 2003.

[41] T. Tagawa, R. Morgan, M. Cristea et al., "Comparison of HMGA2 expression in serous versus endometrioid subtypes of human ovarian carcinoma," in City of Hope Comprehensive Cancer Center, 2010.

[42] L. J. Copeland, L. Vaccarello, and G. S. Lewandowski, "Secondlook laparotomy in epithelial ovarian cancer," Obstetrics and Gynecology Clinics of North America, vol. 21, no. 1, pp. 155166, 1994.

[43] S. C. Rubin, W. J. Hoskins, T. B. Hakes, M. Markman, J. M. Cain, and J. L. Lewis Jr., "Recurrence after negative secondlook laparotomy for ovarian cancer: analysis of risk factors," American Journal of Obstetrics and Gynecology, vol. 159, no. 5, pp. 1094-1098, 1988.

[44] D. K. Armstrong, "Relapsed ovarian cancer: challenges and management strategies for a chronic disease," Oncologist, vol. 7, supplement 5, pp. 20-28, 2002.

[45] M. R. Brown, J. O. Blanchette, and E. C. Kohn, "Angiogenesis in ovarian cancer," Best Practice and Research: Clinical Obstetrics and Gynaecology, vol. 14, no. 6, pp. 901-918, 2000.

[46] L. A. Hefler, A. Mustea, D. Könsgen et al., "Vascular endothelial growth factor gene polymorphisms are associated with prognosis in ovarian cancer," Clinical Cancer Research, vol. 13, no. 3, pp. 898-901, 2007.

[47] R. A. Burger, M. W. Sill, B. J. Monk, B. E. Greer, and J. I. Sorosky, "Phase II trial of bevacizumab in persistent or recurrent epithelial ovarian cancer or primary peritoneal cancer: a Gynecologic Oncology Group study," Journal of Clinical Oncology, vol. 25, no. 33, pp. 5165-5171, 2007.

[48] S. A. Cannistra, U. A. Matulonis, R. T. Penson et al., "Phase II study of bevacizumab in patients with platinum-resistant ovarian cancer or peritoneal serous cancer," Journal of Clinical Oncology, vol. 25, no. 33, pp. 5180-5186, 2007.

[49] ASCO 2011 Annual Meeting Abstracts, http://www.asco.org/ ASCOv2/Meetings/Abstracts? \&vmview=abst_category_ abstracts_view\&confID $=102 \&$ subCatID $=142$.

[50] S. Mabuchi, C. Kawase, D. A. Altomare et al., "mTOR is a promising therapeutic target both in cisplatin-sensitive and cisplatin-resistant clear cell carcinoma of the ovary," Clinical Cancer Research, vol. 15, no. 17, pp. 5404-5413, 2009.

[51] A. Serrano-Olvera, A. Dueñas-González, D. Gallardo-Rincón, M. Candelaria, and J. de la Garza-Salazar, "Prognostic, predictive and therapeutic implications of HER2 in invasive epithelial ovarian cancer," Cancer Treatment Reviews, vol. 32, no. 3, pp. 180-190, 2006.

[52] H. Meden, D. Marx, T. Raab, M. Kron, A. Schauer, and W. Kuhn, "EGF-R and overexpression of the oncogene c-erbB-2 in ovarian cancer: immunohistochemical findings and prognostic value," Journal of Obstetrics and Gynaecology, vol. 21, no. 2, pp. 167-178, 1995.

[53] E. V. Høgdall, L. Christensen, S. K. Kjaer et al., "Distribution of HER-2 overexpression in ovarian carcinoma tissue and its prognostic value in patients with ovarian carcinoma: from the Danish MALOVA ovarian cancer study," Cancer, vol. 98, no. 1, pp. 66-73, 2003.

[54] M. S. Mano, A. Awada, A. di Leo et al., "Rates of topoisomerase II-alpha and HER-2 gene amplification and expression in epithelial ovarian carcinoma," Gynecologic Oncology, vol. 92, no. 3, pp. 887-895, 2004.

[55] Y. Wu, R. A. Soslow, D. S. Marshall, M. Leitao, and B. Chen, "Her-2/neu expression and amplification in early stage ovarian surface epithelial neoplasms," Gynecologic Oncology, vol. 95, no. 3, pp. 570-575, 2004.

[56] D. J. Slamon, W. Godolphin, L. A. Jones et al., "Studies of the HER-2/neu proto-oncogene in human breast and ovarian cancer," Science, vol. 244, no. 4905, pp. 707-712, 1989.

[57] B. Tanner, E. Kreutz, W. Weikel et al., "Prognostic significance of c-erbB-2 mRNA in ovarian carcinoma," Gynecologic Oncology, vol. 62, no. 2, pp. 268-277, 1996.

[58] E. Felip, J. M. del Campo, D. Rubio, M. T. Vidal, R. Colomer, and B. Bermejo, "Overexpression of c-erbB-2 in epithelial ovarian cancer: prognostic value and relationship with response to chemotherapy," Cancer, vol. 75, no. 8, pp. 21472152, 1995.

[59] M. Tuefferd, J. Couturier, F. Penault-Llorca et al., "HER2 status in ovarian carcinomas: a multicenter GINECO study of 320 patients," PLoS ONE, vol. 2, no. 11, Article ID e1138, 2007.

[60] M. S. Gordon, D. Matei, C. Aghajanian et al., "Clinical activity of pertuzumab (rhuMAb 2C4), a HER dimerization inhibitor, in advanced ovarian cancer: potential predictive relationship with tumor HER2 activation status," Journal of Clinical Oncology, vol. 24, no. 26, pp. 4324-4332, 2006.

[61] D. B. Agus, R. W. Akita, W. D. Fox et al., "Targeting ligandactivated ErbB2 signaling inhibits breast and prostate tumor growth," Cancer Cell, vol. 2, no. 2, pp. 127-137, 2002.

[62] J. Konner, R. J. Schilder, F. A. DeRosa et al., "A phase II study of cetuximab/paclitaxel/carboplatin for the initial treatment of advanced-stage ovarian, primary peritoneal, or fallopian tube cancer," Gynecologic Oncology, vol. 110, no. 2, pp. 140-145, 2008.

[63] P. A. Vasey, M. Gore, R. Wilson et al., "A phase Ib trial of docetaxel, carboplatin and erlotinib in ovarian, fallopian tube and primary peritoneal cancers," British Journal of Cancer, vol. 98, no. 11, pp. 1774-1780, 2008. 
[64] H. Hirte, A. Oza, K. Swenerton et al., "A phase II study of erlotinib (OSI-774) given in combination with carboplatin in patients with recurrent epithelial ovarian cancer (NCIC CTG IND.149)," Gynecologic Oncology, vol. 118, no. 3, pp. 308-312, 2010.

[65] S. V. Blank, P. Christos, J. P. Curtin et al., "Erlotinib added to carboplatin and paclitaxel as first-line treatment of ovarian cancer: a phase II study based on surgical reassessment," Gynecologic Oncology, vol. 119, no. 3, pp. 451-456, 2010.

[66] S. E. Rivkin, J. A. M. Carolyn Muller, J. Moon et al., "A phase I/II study of lapatinib plus carboplatin and paclitaxel in relapsed ovarian and breast cancer," Clinical Ovarian Cancer, vol. 2, pp. 112-117, 2009.

[67] A. A. Secord, J. A. Blessing, D. K. Armstrong et al., "Phase II trial of cetuximab and carboplatin in relapsed platinumsensitive ovarian cancer and evaluation of epidermal growth factor receptor expression: a Gynecologic Oncology Group study," Gynecologic Oncology, vol. 108, pp. 493-499, 2008.

[68] "ABT 888 with cytoxan Randomized Phase II study," http://clinicaltrials.gov/ct2/show/NCT01306032 .

[69] F. McPhillips, P. Mullen, K. G. MacLeod et al., "Raf-1 is the predominant Raf isoform that mediates growth factorstimulated growth in ovarian cancer cells," Carcinogenesis, vol. 27, no. 4, pp. 729-739, 2006.

[70] F. McPhillips, P. Mullen, B. P. Monia et al., "Association of cRaf expression with survival and its targeting with antisense oligonucleotides in ovarian cancer," British Journal of Cancer, vol. 85, no. 11, pp. 1753-1758, 2001.

[71] L. F. Sempere, M. Christensen, A. Silahtaroglu et al., "Altered microRNA expression confined to specific epithelial cell subpopulations in breast cancer," Cancer Research, vol. 67, no. 24, pp. 11612-11620, 2007.

[72] F. Yu, H. Yao, P. Zhu et al., "Let-7 regulates self renewal and tumorigenicity of breast cancer cells," Cell, vol. 131, no. 6, pp. 1109-1123, 2007. 


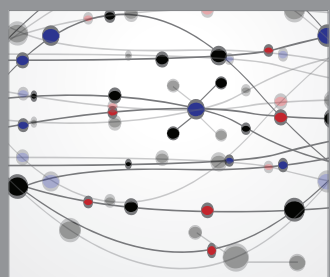

The Scientific World Journal
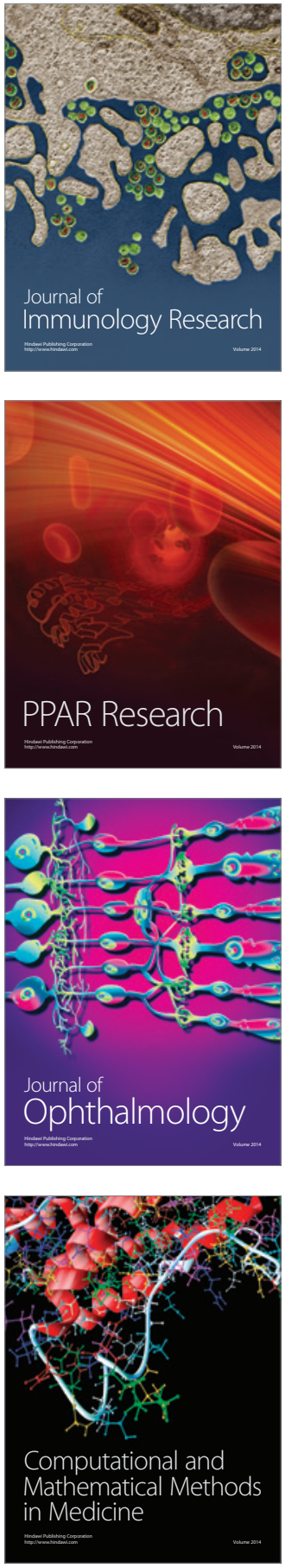

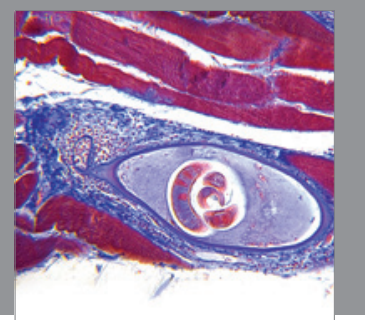

Gastroenterology

Research and Practice
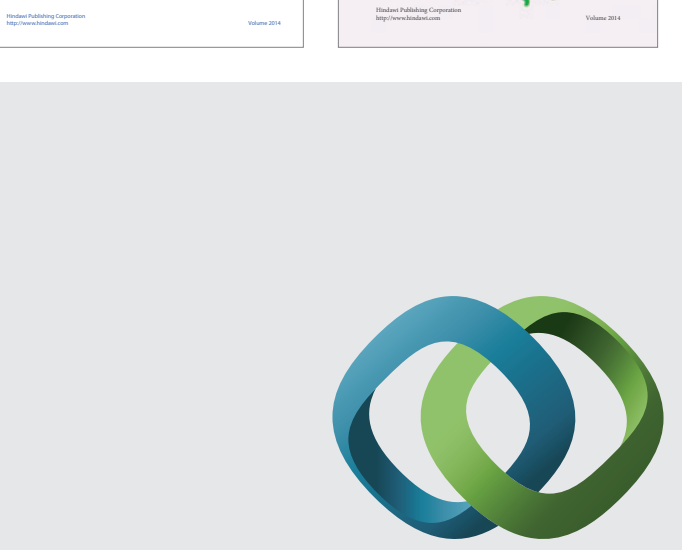

\section{Hindawi}

Submit your manuscripts at

http://www.hindawi.com
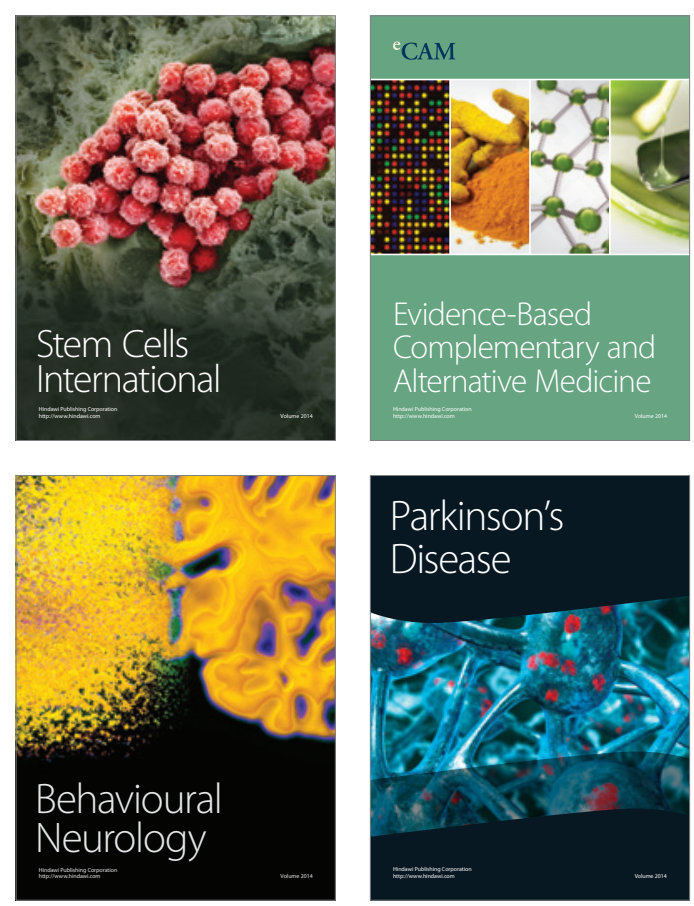

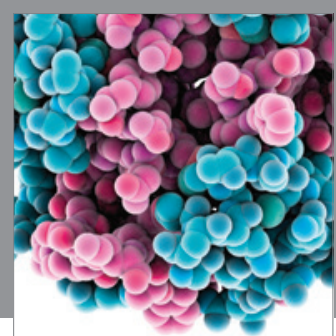

Journal of
Diabetes Research

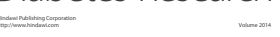

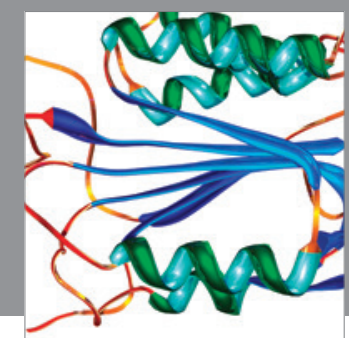

Disease Markers
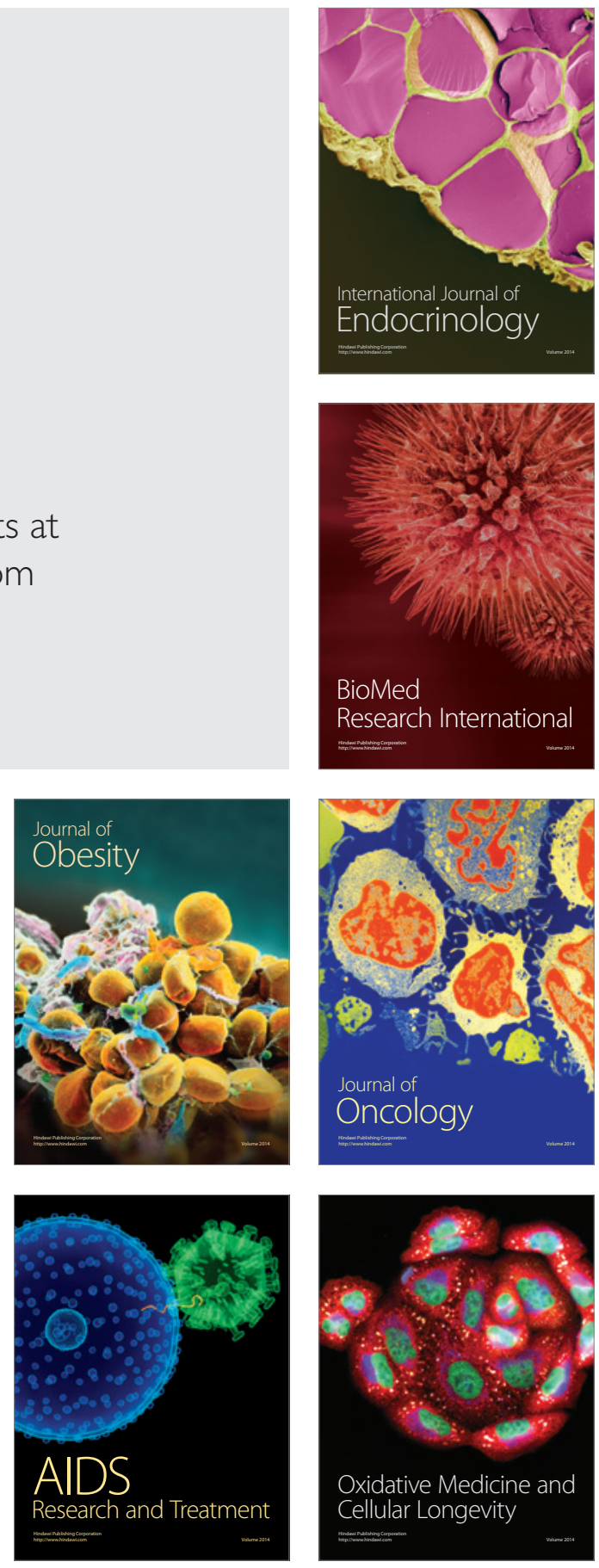